PROCEEDINGS OF THE

AMERICAN MATHEMATICAL SOCIETY

Volume 125, Number 10, October 1997, Pages 3019-3025

S 0002-9939(97)03926-9

\title{
COMBINATORIAL ASPECTS OF $\mathbf{F}_{\sigma}$ FILTERS WITH AN APPLICATION TO $\mathcal{N}$-SETS
}

\author{
CLAUDE LAFLAMME
}

(Communicated by Andreas R. Blass)

\begin{abstract}
We discuss $\mathrm{F}_{\sigma}$ filters and show that the minimum size of a filter base generating an undiagonalizable filter included in some $\mathrm{F}_{\sigma}$ filter is the better known bounded evasion number $\mathfrak{e}_{u b d}$. An application to $\mathcal{N}$-sets from trigonometric series is given by showing that if $A$ is an $\mathcal{N}$-set and $B$ has size less than $\mathfrak{e}_{u b d}$, then $A \cup B$ is again an $\mathcal{N}$-set.
\end{abstract}

\section{INTRODUCTION}

Our terminology is standard but we review the main concepts and notation. The set of natural numbers will be denoted by $\omega, \mathcal{P}(\omega)$ denotes the collection of all its subsets. Given a set $X$, we write $[X]^{\omega}$ and $[X]^{<\omega}$ to denote the collection of infinite or finite subsets of $X$ respectively; if we wish to be more specific, we write $[X]^{n}$ and $[X]^{\leq n}$ to denote the collection of subsets of size $n$ or at most $n$ respectively. We use the well known 'almost inclusion' ordering between members of $[\omega]]^{\omega}$, i.e. $X \subseteq^{*} Y$ if $X \backslash Y$ is finite. We identify $\mathcal{P}(\omega)$ with ${ }^{\omega} 2$ via characteristic functions. The space ${ }^{\omega} 2$ is further equipped with the product topology of the discrete space $\{0,1\}$; a basic neighbourhood is then a set of the form

$$
\mathcal{O}_{s}=\left\{f \in \omega^{\omega}: s \subseteq f\right\}
$$

where $s \in<\omega^{2}$, the collection of finite binary sequences. The terms "nowhere dense", "meager", "Baire property" and " $\mathrm{F}_{\sigma}$ " all refer to this topology. We also write ${ }^{\omega} \omega$ to denote all functions on the natural numbers. The ordering of eventual dominance is defined by $f \leq^{*} g$ if $f(n) \leq g(n)$ for all but finitely many $n$. Without further mention, terminology with respect to families of functions all refer to this ordering; in particular a family $\mathcal{H} \subseteq \omega_{\omega}$ is said to be bounded if it is bounded by a single function in this ordering.

A filter is a collection of subsets of $\omega$ containing all cofinite sets and closed under finite intersections and supersets. It is called proper if it does not contain the empty set; thus the collection of cofinite sets is the smallest proper filter, it is called the Fréchet filter and is denoted by $\mathfrak{F} r$. To avoid trivialities, we shall assume that all filters under discussion are proper. An infinite set $X \in[\omega]^{\omega}$ is said to zap (or diagonalize) a filter $\mathcal{F}$ if $X \subseteq^{*} Y$ for each $Y \in \mathcal{F}$. Given a collection of sets

Received by the editors September 18, 1995 and, in revised form, May 1, 1996.

1991 Mathematics Subject Classification. Primary 04A20; Secondary 03E05, 03E15, 03E35.

This research was partially supported by NSERC of Canada.

(C) 1997 American Mathematical Society 
$\mathcal{X} \subseteq[\omega]^{\omega}$, we denote by $\langle\mathcal{X}\rangle$ the filter generated by $\mathcal{X}$, that is, the smallest filter containing each member of $\mathcal{X}$.

The Katětov ordering on filters is defined by

$$
\mathcal{F} \leq_{K} \mathcal{G} \text { if }\left(\exists f \in \omega_{\omega}\right) \mathcal{G} \supseteq\left\{f^{-1}\{X\}: X \in \mathcal{F}\right\} .
$$

The following Lemma from [9] combinatorially describes $\mathrm{F}_{\sigma}$ filters.

Lemma 1.1. Let $\mathcal{F}$ be an $F_{\sigma}$ filter and $g \in \omega_{\omega}$. Then there is an increasing sequence of natural numbers $\left\langle n_{k}: k \in \omega\right\rangle$ and sets $a_{i}^{k} \subseteq\left[n_{k}, n_{k+1}\right), i<m_{k}$, such that

(1) $\left(\forall x \in\left[m_{k}\right]^{\leq g(k)}\right) \bigcap_{i \in x} a_{i}^{k} \neq \emptyset$,

(2) $(\forall X \in \mathcal{F})\left(\forall^{\infty} k\right)\left(\exists i<m_{k}\right) a_{i}^{k} \subseteq X$.

Proof. Let $\mathcal{F}=\bigcup_{n} \mathcal{C}_{n}$ where each $\mathcal{C}_{n}$ is closed and put $\mathcal{C}=\{X \cup n: n \in \omega$ and $X \in$ $\left.\mathcal{C}_{n}\right\}$. Then again $\mathcal{C}$ is a closed set and every member of $\mathcal{F}$ is almost equal to a member of $\mathcal{C}$.

Let $n_{0}=0$ and having defined $n_{j}$ for $j \leq k$, choose an $n_{k+1}>n_{k}$ such that

$$
\left(\forall X_{0}, X_{1}, \cdots, X_{g(k)-1} \in \mathcal{C}\right) \bigcap_{i<g(k)} X_{i} \cap\left[n_{k}, n_{k+1}\right) \neq \emptyset .
$$

The existence of such an $n_{k+1}$ follows from the fact that $\mathcal{C}$ is closed and that $\mathcal{F}$ only contains infinite sets. Now enumerate $\left\{X \cap\left[n_{k}, n_{k+1}\right): X \in \mathcal{C}\right\}$ as $\left\{a_{i}^{k}: i<\right.$ $\left.m_{k}\right\}$ and this completes the proof.

It is worth noticing that conversely, given a family $\left\langle\left\langle a_{i}^{k}: i\left\langle m_{k}\right\rangle: k \in \omega, g\right\rangle\right.$ satisfying conditions (1) and (2) above, then the collection

$$
\left\{X:(\forall k)\left(\exists i<m_{k}\right) a_{i}^{k} \subseteq X\right\}
$$

is a closed set generating an $\mathrm{F}_{\sigma}$ filter whenever $\lim _{n} g(n)=\infty$.

We thank Andreas Blass, Jörg Brendle, Juris Steprāns and the referee for valuable comments on the paper.

\section{2. $\mathrm{F}_{\sigma}$ FiLters that CANNOT BE ZAPPED}

We first present a combinatorial description of the smallest size of a family of sets generating a filter that cannot be zapped but which is included in some $\mathrm{F}_{\sigma}$ filter. This is a variation of some well known cardinals; indeed the cardinal $\mathfrak{p}$ is defined as the smallest size of a family of sets generating a filter that cannot be zapped and $\mathfrak{t}$ is defined as the smallest size of a well ordered (under almost inclusion) family of sets generating a filter that cannot be zapped. It turns out that these cardinals have a substantial impact on the set theory of the reals.

\section{Definition 2.1.}

$\mathfrak{f}=\min \{|\mathcal{X}|: \mathcal{X}$ generates a filter that cannot be zapped but which is included in some $\mathrm{F}_{\sigma}$ filter $\}$.

$\mathfrak{f}_{1}=\min \left\{|\mathcal{H}|: \mathcal{H} \subseteq \omega_{\omega}\right.$ is bounded and for some $g \in \omega_{\omega}$ with $\lim _{n \rightarrow \infty} g(n)=\infty$, $\left.\left(\forall X \in[\omega]^{\omega}\right)\left(\forall s_{n} \in[\omega]^{\leq g(n)}\right)(\exists h \in \mathcal{H})\left(\exists^{\infty} n \in X\right) h(n) \notin s_{n}.\right\}$

$\mathfrak{f}_{2}=\min \left\{|\mathcal{H}|: \mathcal{H} \subseteq \omega_{\omega}\right.$ is bounded and for some $g \in \omega_{\omega}$ with $\lim _{n \rightarrow \infty} g(n)=\infty$, $\left(\forall X \in[\omega]^{\omega}\right)\left(\forall \pi_{n}: n^{\omega} \rightarrow[\omega] \leq g(n)\right)(\exists h \in \mathcal{H})\left(\exists^{\infty} n \in X\right) h(n) \notin \pi_{n}(h\lceil n)$. 
$\mathfrak{e}_{u b d}=\min \left\{|\mathcal{H}|: \mathcal{H} \subseteq \omega_{\omega}\right.$ is bounded and

$$
\left(\forall X \in[\omega]^{\omega}\right)\left(\forall \pi_{n}: n_{\omega} \rightarrow \omega\right)(\exists h \in \mathcal{H})\left(\exists^{\infty} n \in X\right) h(n) \neq \pi_{n}(h\lceil n) .\}
$$

The cardinal $\mathfrak{e}_{u b d}$ is due to Brendle [5], and Brendle and Shelah [6]. Eisworth has shown (unpublished) that $\mathfrak{e}_{u b d} \leq \mathfrak{f}$ and an argument very similar to that of Blass in [4] shows that $\mathfrak{f}_{1} \leq \mathfrak{e}_{u b d}$. We extend these results by showing that all four cardinals are equal.

Proposition 2.2. The four cardinals $\mathfrak{f}, \mathfrak{f}_{1}, \mathfrak{f}_{2}$ and $\mathfrak{e}_{u b d}$ are equal.

Proof. For simplicity, we prove $\mathfrak{f} \leq \mathfrak{f}_{1} \leq \mathfrak{e}_{u b d} \leq \mathfrak{f}_{2} \leq \mathfrak{f}_{1} \leq \mathfrak{f}$.

$\mathfrak{f} \leq \mathfrak{f}_{1}$ : Let $\mathcal{H} \subseteq \omega_{\omega}$ be given of size $|\mathcal{H}|<\mathfrak{f}$, without loss of generality bounded everywhere by $b \in \omega_{\omega}$, and fix $g \in \omega_{\omega}$ such that $\lim _{n} g(n)=\infty$.

Consider $\mathcal{A}_{n}=[b(n)]^{\leq g(n)}$ and for $i<b(n)$, put $a_{i}^{n}=\left\{x \in \mathcal{A}_{n}: i \in x\right\}$. Notice that

$$
\left(\forall x \in \mathcal{A}_{n}=[b(n)]^{\leq g(n)}\right) \bigcap_{i \in x} a_{i}^{n} \neq \emptyset .
$$

Identify $\bigcup_{n} \mathcal{A}_{n}$ with $\omega$, and form the filter $\mathcal{F}$ generated by

$$
\left\{\bigcup_{n} a_{h(n)}^{n}: h \in \mathcal{H}\right\} .
$$

Then $\mathcal{F}$ is a filter generated by fewer than $\mathfrak{f}$ sets and included in the $\mathrm{F}_{\sigma}$ filter $\left\langle\left\langle a_{i}^{n}: i\langle b(n)\rangle: n \in \omega, g\right\rangle\right.$. Therefore $\mathcal{F}$ must be zapped, which means here that for some $X \in[\omega]^{\omega}$ and $x_{n} \in \mathcal{A}_{n}$ for $n \in X,\left\{x_{n}: n \in X\right\}$ zaps $\mathcal{F}$. In particular, for $h \in \mathcal{H}$,

$$
\left\{x_{n}: n \in X\right\} \subseteq^{*}\left\{a_{h(n)}^{n}: n \in \omega\right\}
$$

and thus $h(n) \in x_{n}$ for all but finitely many $n \in X$.

$\mathfrak{f}_{1} \leq \mathfrak{e}_{u b d}$ : Let $\mathcal{H} \subseteq \omega_{\omega}$ be given of size $|\mathcal{H}|<\mathfrak{f}_{1}$, without loss of generality bounded everywhere by $b \in \omega_{\omega}$. Partition $\omega$ into consecutive intervals $\left\langle\mathcal{I}_{n}=\right.$ $\left.\left[a_{n}, a_{n+1}\right): n \in \omega\right\rangle$ such that $a_{n+1}-a_{n}>n^{2}$.

For $h \in \mathcal{H}$, define $\tilde{h}(n)=h\left\lceil\mathcal{I}_{n}\right.$ and form $\tilde{\mathcal{H}}=\{\tilde{h}: h \in \mathcal{H}\}$.

Identifying $\prod_{a_{n} \leq i<a_{n+1}} b(i)$ with its cardinality, we have that $\tilde{\mathcal{H}}$ is a bounded family of size $|\tilde{\mathcal{H}}|<\mathfrak{f}_{1}$. Therefore there are $\tilde{X} \in[\omega]^{\omega}$ and $s_{n} \in\left[\prod_{a_{n} \leq i<a_{n+1}} b(i)\right]^{\leq n}$ such that

$$
(\forall \tilde{h} \in \tilde{\mathcal{H}})\left(\forall^{\infty} n \in \tilde{X}\right) \tilde{h}(n) \in s_{n} .
$$

Now by the pigeonhole principle, there must be for each $n \in \tilde{X}$ an $i_{n} \in \mathcal{I}_{n}$ such that

$$
(*) \quad\left(\forall t, t^{\prime} \in s_{n}\right) t\left\lceil i_{n}=t^{\prime}\left\lceil i_{n} \rightarrow t\left(i_{n}\right)=t^{\prime}\left(i_{n}\right) ;\right.\right.
$$

this is where we use the fact that $\left|\mathcal{I}_{n}\right|>n^{2}$ while $\left|s_{n}\right| \leq n$.

Let $X=\left\{i_{n}: n \in \tilde{X}\right\}$ and define $\pi_{i}:{ }^{i} \omega \rightarrow \omega$ as follows. If $i=i_{n} \in X$ and $t \in{ }^{i} \omega$ is such that $t\left\lceil\left[a_{n}, i\right)\right.$ is an initial segment of a member $t^{\prime}$ of $s_{n}$, then define $\pi_{i}(t)=t^{\prime}\left(i_{n}\right)$; this is well defined by the choice of $i_{n}$. In all other cases define $\pi_{i}(t)$ arbitrarily.

Now for $h \in \mathcal{H}, \tilde{h} \in s_{n}$ for all but finitely many $n \in \tilde{X}$; for each such $n$, $i=i_{n} \in X, h \uparrow\left[a_{n}, i\right)$ is an initial segment of a member of $s_{n}$, namely $\tilde{h}(n)=h\left\lceil\mathcal{I}_{n}\right.$, and thus $\pi_{i}\left(h\lceil i)=h(i)\right.$. This proves that $\mathfrak{f}_{1} \leq \mathfrak{e}_{u b d}$ as desired.

$\mathfrak{e}_{\boldsymbol{u b d}} \leq \mathfrak{f}_{2}$ : This inequality is trivial.

$\mathfrak{f}_{2} \leq \mathfrak{f}_{1}$ : Let $\mathcal{H} \subseteq \omega_{\omega}$ be given of size $|\mathcal{H}|<\mathfrak{f}_{2}$, without loss of generality bounded everywhere by $b \in \omega_{\omega}$, and fix $g \in \omega_{\omega}$ such that $\lim _{n} g(n)=\infty$. 
Choose integers $\delta_{0}=0<\delta_{1}<\ldots$ such that

$$
(\forall n) \prod_{i \leq \delta_{n}} b(i) \times g\left(\delta_{n}\right) \leq g\left(\delta_{n+1}\right) .
$$

Now for $n \in \omega$, define

$$
\tilde{b}(n)=\prod_{\delta_{n} \leq i \leq \delta_{n+1}} b(i)
$$

which we identify with the cartesian product. For $h \in \mathcal{H}$, define

$$
\tilde{h}(n)=h \uparrow\left[\delta_{n}, \delta_{n+1}\right] \in \tilde{b}(n)
$$

and put $\tilde{\mathcal{H}}=\{\tilde{h} ; h \in \mathcal{H}\}$, a bounded family of size less than $\mathfrak{f}_{2}$. Therefore

$$
\left(\exists \tilde{X} \in[\omega]^{\omega}\right)\left(\exists \pi_{n}:{ }^{n} \omega \rightarrow[\tilde{b}(n)]^{\leq g\left(\delta_{n}\right)}\right)(\forall \tilde{h} \in \tilde{\mathcal{H}})\left(\forall^{\infty} n \in \tilde{X}\right) \tilde{h}(n) \in \pi_{n}(\tilde{h}\lceil n) .
$$

For $n \in \tilde{X}$, let

$$
s_{\delta_{n+1}}=\left\{u\left(\delta_{n+1}\right): u \in \pi_{n}(t) \text { for } t \in \prod_{i<n} \tilde{b}(i)\right\}
$$

Then

$$
\left|s_{\delta_{n+1}}\right| \leq \prod_{i \leq \delta_{n}} b(i) \times g\left(\delta_{n}\right) \leq g\left(\delta_{n+1}\right),
$$

and so $s_{\delta_{n+1}} \in\left[b\left(\delta_{n+1}\right)\right] \leq g\left(\delta_{n+1}\right)$. Finally, given $h \in \mathcal{H}$, and thus $\tilde{h} \in \tilde{\mathcal{H}}$,

$$
\left(\forall^{\infty} n \in \tilde{X}\right) \tilde{h}(n) \in \pi_{n}(\tilde{h}\lceil n),
$$

and so

$$
\left(\forall^{\infty} n \in \tilde{X}\right) h\left(\delta_{n+1}\right) \in s_{\delta_{n+1}} .
$$

Since $g$ was arbitrary, $|\mathcal{H}|<\mathfrak{f}_{1}$ and we conclude that $\mathfrak{f}_{2} \leq \mathfrak{f}_{1}$.

$\mathfrak{f}_{1} \leq \mathfrak{f}$ : Let $\mathcal{F}$ be a filter generated by $\left\langle A_{\alpha}: \alpha<\kappa\right\rangle, \kappa<\mathfrak{f}_{1}$, and included in the $\mathrm{F}_{\sigma}$ filter $\left\langle\left\langle a_{i}^{k}: i<m_{k}\right\rangle: k \in \omega, g\right\rangle$.

For each $\alpha<\kappa$, define a function $f_{\alpha} \in \omega_{\omega}$ such that for all but finitely many $k$, $a_{f_{\alpha}(k)}^{k} \subseteq A_{\alpha}$.

Then $\left\{f_{\alpha}: \alpha<\kappa\right\}$ is a bounded family of size less than $\mathfrak{f}_{1}$, and therefore

$$
\left(\exists X \in[\omega]^{\omega}\right)\left(\exists s_{k} \in\left[m_{k}\right]^{\leq g(k)}\right)(\forall \alpha)\left(\forall^{\infty} k \in X\right) \quad f_{\alpha}(k) \in s_{k} .
$$

We conclude that $\bigcup_{k \in X} \bigcap_{i \in s_{k}} a_{i}^{k}$ zaps the filter $\mathcal{F}$.

We conclude this section by giving a small perspective on these cardinals (see [12] for a description of cardinals not defined here). If one removes the boundedness restriction on $\mathcal{H}$, the cardinal $\mathfrak{e}_{u b d}$ becomes the evasion number, known as $\mathfrak{e}([4])$; clearly $\mathfrak{e} \leq \mathfrak{e}_{u b d}$ and it has been proved consistent by Shelah that $\mathfrak{e}<\mathfrak{e}_{u b d}[6]$. Removing the boundedness restriction on $\mathcal{H}$ and fixing $X=\omega$, the cardinal $\mathfrak{f}_{1}$ is the additivity of measure, $\operatorname{add}(\mathcal{N})([1])$; keeping the boundedness and using $X=\omega$ yields the so-called transitive additivity of measure (due to Pawlikovski), and finally removing the boundedness condition but keeping $X$ arbitrary yields the cardinal $\mathfrak{s} e$ (see [4]). Thus we have $\operatorname{add}(\mathcal{N}) \leq \mathfrak{e} \leq \mathfrak{e}_{u b d}=\mathfrak{f}$, and $\mathfrak{s e}$,trans $-\operatorname{add}(\mathcal{N}) \leq \mathfrak{e}_{u b d}$.

For upper bounds, it is already known that $\mathfrak{e}_{u b d}$ is less than or equal to the uniformity of the null and meager ideals; these are easy to prove through the cardinal $\mathfrak{f}_{1}$.

It is also known that the cardinal $\mathfrak{e}_{u b d}$ is not provably equal to any of the standard cardinals $\mathfrak{b}, \mathfrak{d}, \mathfrak{t}$ or additivity, uniformity, cofinality and covering of the null or meager ideals (see [9], [11], [6]). 
A different but provable lower bound however for the number $\mathfrak{e}_{u b d}$ (and thus $\mathfrak{f}$ ) is $\mathfrak{t}$; the idea of the proof is from [2]. We shall use the cardinal $\mathfrak{b}$, the minimum size of an unbounded family in $\omega_{\omega}$, and the well-known inequality $\mathfrak{t} \leq \mathfrak{b}$ (see [12]).

Brendle remarks that from $\mathfrak{t} \leq \mathfrak{e}_{u b d}$ and his result with Shelah $([5,6])$ that $\mathfrak{s} e=\min \{\mathfrak{e}, \mathfrak{b}\}=\min \left\{\mathfrak{e}_{u b d}, \mathfrak{b}\right\}$, one concludes that $\mathfrak{t} \leq \mathfrak{s} e$, thus improving Blass' result $\mathfrak{p} \leq \mathfrak{s e}[4]$. Brendle also noticed that this can be proved directly from the following result as any family of functions of size less than $t$ is necessarily bounded.

Proposition 2.3. $\mathfrak{t} \leq \mathfrak{e}_{u b d} \leq 2^{\aleph_{0}}$.

Proof. We show that $\mathfrak{t} \leq \mathfrak{f}_{1}$. Let $\mathcal{H}=\left\langle h_{\alpha}: \alpha<\kappa\right\rangle$ be a family of size $\kappa<\mathfrak{t}$, bounded everywhere by $b \in \omega_{\omega} \omega$, and fix $g \in \omega_{\omega}$ such that $\lim _{n} g(n)=\infty$.

We construct a sequence $\left\langle\phi_{\alpha}: \alpha \leq \kappa\right\rangle$ such that:

(1) $\phi_{\alpha}: \operatorname{dom}\left(\phi_{\alpha}\right) \rightarrow[\omega]^{<\omega}$,

- $\operatorname{dom}\left(\phi_{\alpha}\right) \in[\omega]^{\omega}$,

- $\phi_{\alpha}(k) \in[b(k)]^{\leq g(k)}$,

- $\lim _{k \in \operatorname{dom}\left(\phi_{\alpha}\right)} g(k)-\left|\phi_{\alpha}(k)\right|=+\infty$,

(2) $(\forall \beta<\alpha) \operatorname{dom}\left(\phi_{\alpha}\right) \subseteq \subseteq^{*} \operatorname{dom}\left(\phi_{\beta}\right)$, and $\left(\forall^{\infty} k \in \operatorname{dom}\left(\phi_{\alpha}\right)\right) \phi_{\beta}(k) \subseteq \phi_{\alpha}(k)$,

(3) $(\forall \beta<\alpha)\left(\forall^{\infty} k \in \operatorname{dom}\left(\phi_{\alpha}\right)\right) h_{\beta}(k) \in \phi_{\alpha}(k)$.

Once we have obtained $\phi_{\kappa}$, then clearly $s_{k}=\phi_{\kappa}(k)$ for $k \in \operatorname{dom}\left(\phi_{\kappa}\right)$ is as desired.

Now to construct the sequence, assume that we already have $\left\{\phi_{\beta}: \beta<\alpha\right\}$ for some $\alpha \leq \kappa$.

If $\alpha=\beta+1$ is a successor ordinal, define $\phi_{\alpha}(k)=\phi_{\beta}(k) \cup\left\{h_{\alpha}(k)\right\}$ for $k \in \operatorname{dom}\left(\phi_{\beta}\right)$ such that $\left|\phi_{\beta}(k)\right|<g(k)$.

For $\alpha$ a limit ordinal, first choose a $\tilde{g} \in \omega_{\omega} \omega$ such that $\lim _{k} g(k)-\tilde{g}(k)=\infty$ and

$$
(\forall \beta<\alpha)\left(\forall^{\infty} k \in \operatorname{dom}\left(\phi_{\beta}\right)\right)\left|\phi_{\beta}(k)\right| \leq \tilde{g}(k) .
$$

This is possible as $\alpha \leq \kappa<\mathfrak{t} \leq \mathfrak{b}$; I am not sure where this idea comes from.

Now for $\beta<\alpha$ define

$$
A_{\beta}=\left\{\langle k, x\rangle: k \in \operatorname{dom}\left(\phi_{\beta}\right) \text { and } \phi_{\beta}(k) \subseteq x \subseteq b(k) \text { and }|x| \leq \tilde{g}(k)\right\} .
$$

Clearly $\beta<\gamma<\alpha \Longrightarrow A_{\gamma} \subseteq^{*} A_{\beta}$ and, identifying $A_{0}$ with $\omega$ and as $\alpha<\mathfrak{t}$, we can find some infinite $A_{\alpha} \subseteq^{*} A_{\beta}$ for each $\beta<\alpha$. Finally we put

$$
\phi_{\alpha}(k)=\text { any } x \text { such that }\langle k, x\rangle \in A_{\alpha}
$$

and undefined if there is no such $x$. Clearly $\phi_{\alpha}$ is as desired.

\section{Trigonometric SERIES AND $\mathcal{N}$-SETS}

In this section we prove that if $A$ is an $\mathcal{N}$-set and $|B|<\mathfrak{e}_{u b d}$, then $A \cup B$ is also an $\mathcal{N}$-set. A similar result was earlier proved with $\mathfrak{p}$ in the role of $\mathfrak{e}_{u b d}$ in [7] on which we modeled our proof, and then with $\mathfrak{t}$ in [2] on which we modeled the above proof of $\mathfrak{t} \leq \mathfrak{e}_{u b d}$.

It is known that the collection of $\mathcal{N}$-sets is not in general closed under unions; in [3] two $\mathcal{N}$-sets of cardinality $\mathfrak{c}$ are constructed whose union is not an $\mathcal{N}$-set.

Definition 3.1. A set $A \subseteq \mathbb{R}$ is called an $\mathcal{N}$-set ([3]) if there is a sequence of non-negative reals $\left\langle a_{n}: n \in \omega\right\rangle$ such that:

(1) $\sum_{n=0}^{\infty} a_{n}=+\infty$,

(2) $\quad(\forall a \in A) \sum_{n=0}^{\infty} a_{n}|\sin \pi n a|<\infty$. 
Proposition 3.2. If $A \subseteq \mathbb{R}$ is an $\mathcal{N}$-set and $|B|<\mathfrak{e}_{u b d}$, then $A \cup B$ is also an $\mathcal{N}$-set.

Proof. Fix a sequence of nonnegative reals $\left\langle a_{n}: n \in \omega\right\rangle$ as in the definition for the $\mathcal{N}$-set $A$. As is now standard procedure (see [7] and [2]), we put $s_{n}=\sum_{i=0}^{n} a_{i}$ and $b_{n}=a_{n} / s_{n}$; then again $\sum_{n=0}^{\infty} b_{n}=+\infty$.

As in [3], find an unbounded, nondecreasing sequence of natural numbers $\left\langle q_{n}\right.$ : $n \in \omega\rangle$ such that

$$
\sum_{n=0}^{\infty} a_{n} / s_{n}^{1+\frac{1}{q_{n}}}<\infty
$$

as we may replace the sequence $\left\langle q_{n}: n \in \omega\right\rangle$ by any slower but unbounded and monotonic sequence, we may as well assume that $q_{\pi_{n}} \leq n$. Let $\epsilon_{n}=s_{n}^{-1 / q_{n}}$.

Choose an increasing sequence of natural numbers $\left\langle\pi_{n}: n \in \omega\right\rangle$ such that

(1) $(\forall k) \sum_{i=\pi_{k}}^{\pi_{k+1}-1} b_{i} \geq 1$,

(2) $(\forall n)\left(\forall m \geq \pi_{n}\right) q_{m} \geq n^{2}$.

Finally for $T \subseteq B$ and $m \in \omega$, define

$$
a_{T}^{m}=\left\{k \in \omega: 0 \leq k \leq s_{m} \text { and }(\forall t \in T)|\sin \pi k m t| \leq 2 \pi \epsilon_{m}\right\},
$$

and for each $n, m \geq \pi_{n}$ and $|T| \leq q_{\pi_{n}} / n$, put

$$
b_{T}^{m}=\left\{a_{T^{\prime}}^{m}: T \subseteq T^{\prime} \text { and }\left|T^{\prime}\right| \leq q_{\pi_{n}}\right\} .
$$

The following claim is from standard number theory and follows from the pigeon hole principle.

Claim 3.3. If $T \subseteq B$ and $|T| \leq q_{\pi_{n}}$, then $a_{T}^{m} \neq \emptyset$ for any $m \geq \pi_{n}$.

Proof. Fix $n, m$ and we let $T=\left\{t_{i}: i<|T|\right\}$. Define a map

$$
c:\left(1 / \epsilon_{m}\right)^{|T|}+1 \rightarrow \prod_{i \in|T|} 1 / \epsilon_{m}
$$

by $c(j)=\left(\ell_{0}, \cdots, \ell_{|T|-1}\right)$ if

$$
(\forall i<|T|)(\exists p \in \omega) p+\ell_{i} \epsilon_{m} \leq j m t_{i}<p+\left(\ell_{i}+1\right) \epsilon_{m} .
$$

There must then be two integers $j_{1}<j_{2}<\left(1 / \epsilon_{m}\right)^{|T|}+1$ such that $c\left(j_{1}\right)=c\left(j_{2}\right)$ and let $k=j_{2}-j_{1}$.

Then $k \leq\left(1 / \epsilon_{m}\right)^{|T|} \leq\left(1 / \epsilon_{m}\right)^{q_{\pi_{n}}} \leq\left(1 / \epsilon_{m}\right)^{q_{m}}=s_{m}$. Now using $\llbracket x \rrbracket$ to denote the distance from $x$ to the nearest integer, we have, for $t \in T$,

$$
|\sin \pi k m t| \leq \pi \llbracket k m t \rrbracket \leq 2 \pi \epsilon_{m}
$$

as desired.

Therefore $a_{T^{\prime}}^{m} \neq \emptyset$ for each $a_{T^{\prime}}^{m} \in b_{T}^{m}$ and of course, if $T_{i} \subseteq B, i<n$, and each $\left|T_{i}\right| \leq q_{\pi_{n}} / n$, then $\bigcap_{i<n} b_{T_{i}}^{m} \neq \emptyset$ whenever $m \geq \pi_{n}$.

Now consider the set

$$
\mathcal{W}=\left\{\left\langle a_{T_{m}}^{m}: \pi_{n} \leq m<\pi_{n+1}\right\rangle: n \in \omega \text { and }\left|T_{m}\right| \leq q_{\pi_{n}}\right\},
$$

which we may identify with $\omega$ since it is a countable set, and the filter

$$
\begin{aligned}
\mathcal{G}= & \left\langle\left\{ X \subseteq \mathcal{W}:(\forall n)\left(\forall \pi_{n} \leq m<\pi_{n+1}\right)\right.\right. \\
& \left.\left.\left(\exists T_{m} \subseteq B\right)\left|T_{m}\right| \leq q_{\pi_{n}} / n \text { and } \prod_{\pi_{n} \leq m<\pi_{n+1}} b_{T_{n}}^{m} \subseteq X\right\}\right\rangle .
\end{aligned}
$$


Then $\mathcal{G}$ is an $\mathrm{F}_{\sigma}$ filter as it is generated by a closed set and contains the filter

$$
\begin{aligned}
\mathcal{F}= & \langle\{X \subseteq \mathcal{W}:(\exists T \subseteq B)|T|<\infty \\
& \left.\left.\quad \text { and for all } n \text { such that }|T| \leq q_{\pi_{n}} / n, \prod_{\pi_{n} \leq m<\pi_{n+1}} b_{T}^{m} \subseteq X\right\}\right\rangle .
\end{aligned}
$$

As $\mathcal{F}$ is generated by fewer than $\mathfrak{e}_{u b d}=\mathfrak{f}$ sets, it must be diagonalized by an infinite set $\mathcal{X} \subseteq \mathcal{W}$ which, without loss of generality, is of the form

$$
\mathcal{X}=\left\{\left\langle a_{T_{m}}^{m}: \pi_{n_{\ell}} \leq m<\pi_{n_{\ell+1}-1}\right\rangle: \ell \in \omega\right\}
$$

where $\left|T_{m}\right| \leq q_{\pi_{n_{\ell}}}$ and $n_{\ell}<n_{\ell+1}$. For each $\ell$ and $\pi_{n_{\ell}} \leq m<\pi_{n_{\ell+1}-1}$, pick $k_{m} \in a_{T_{m}}^{m}$.

Now clearly $\sum_{\ell} \sum_{\pi_{n_{\ell}} \leq m<\pi_{n_{\ell+1}-1}} b_{m}=\infty$ and it remains to show that

$$
\sum_{\ell} \sum_{\pi_{n_{\ell}} \leq m<\pi_{n_{\ell+1}-1}} b_{m}\left|\sin m k_{m} \pi x\right|<\infty
$$

for each $x \in A \cup B$. For $x \in A$,

$$
b_{m}\left|\sin m k_{m} \pi x\right| \leq b_{m} k_{m}|\sin m \pi x| \leq b_{m} s_{m}|\sin m \pi x|=a_{m}|\sin m \pi x| .
$$

Finally for $x \in B$, let $T=\{x\}$ and thus for all but finitely many $\ell$, for all $\pi_{n_{\ell}} \leq$ $m<\pi_{n_{\ell+1}-1}$,

$$
\left|\sin m k_{m} \pi x\right| \leq 2 \pi \epsilon_{m}
$$

and therefore

$$
b_{m}\left|\sin m k_{m} \pi x\right| \leq 2 \pi a_{m} / s_{m}^{1+1 / q_{m}} .
$$

This completes the proof.

\section{REFERENCES}

1. T. Bartoszyński, Combinatorial Aspects of Measure and Category, Fund. Math. 127 (1987), 225-239. MR 88m:04001

2. T. Bartoszyński and M. Scheepers, Remarks on Sets related to Trigonometric Series, Topology and Appl. 64 (1995), 133-140. MR 96h:42004

3. N.K. Bary, A Treatise on Trigonometric Series, MacMillan, New York, 1964. MR 30:1347

4. A. Blass, Cardinal Characteristics and the Product of Countably many Infinite Cyclic groups, Journal of Algebra 169 (1994), 512-540. MR 95h:20069

5. J. Brendle, Evasion and Prediction, the Specker Phenomenon and Gross Spaces, Forum Math. 7 (1995), no. 5, 513-541. MR 96i:03042

6. J. Brendle and S. Shelah, Evasion and Prediction II, J. London Math. Soc. (2) 53 (1996), no. 1, 19-27. CMP 96:04

7. Z. Bukovská and L. Bukovský, Adding Small Sets to an N-set, Proc. Amer. Math. Soc. (to appear, 1994)

8. M. Daguenet, Emploi des Filtres sur $\mathbb{N}$ dans l'Etude Descriptive des Fonctions, Fund. Math. XCV (1977), 11-33. MR 57:10671

9. C. Laflamme, Zapping Small Filters, Proc. Amer. Math. Soc. 114 (1992), 535-544. MR 92e:03076

10. S. Shelah, Proper Forcing, Lecture Notes in Mathematics, Springer-Verlag, Berlin 1982. MR 84h:03002

11. S. Shelah, Vive la Différence I, Set Theory of the Continuum (H. Judah, W. Just and H. Woodin, eds.), Mathematical Sciences Research Institute Publications, Springer-Verlag, 1992, 357-405. MR 94g:03068

12. J. Vaughan, Small Uncountable Cardinals and Topology, Open problems in Topology (J. van Mill and G. Reed, eds.), North-Holland, 1990, 195-218. CMP 91:03

Department of Mathematics and Statistics, University of Calgary, Calgary, AlBerta, CANADA T2N 1N4

E-mail address: laflamme@acs.ucalgary.ca 